\title{
Digital Holography-based Quantitative Elastography using Noise-Correlation
}

\author{
Agathe Marmin $^{1}$, Simon Chatelin ${ }^{1}$, Manuel Flury ${ }^{1}$, Sybille Facca ${ }^{1,2}$, Stefan Catheline ${ }^{3}$, Amir Nahas ${ }^{1}$ \\ ${ }^{1}$ ICUBE Research Institute, The University of Strasbourg, 1 place de l'Hôpital, 67000 Strasbourg, France \\ ${ }^{2}$ Department of Hand Surgery, SOS hand, University Hospital of Strasbourg, FMTS, ICube CNRS 7357, University of Strasbourg, 1 avenue \\ Molière, 67000, Strasbourg, France \\ ${ }^{3}$ LabTAU, Inserm U1032, 151 cours Albert Thomas, 69003 Lyon, France \\ amarmin@unistra.fr
}

\begin{abstract}
Quantitative elastography is performed using noise-correlation on full-field images acquired using digital holography. Experimental results isotropic and anisotropic polymer samples are presented as well as stiffness images on biological tissues.
\end{abstract}

(C) 2021 A. Marmin, S. Chatelin, M. Flury, S. Facca, S. Catheline, A. Nahas

Palpation has been used since the very beginning of medicine to diagnostic pathologies using tissues stiffness. Indeed, mechanical properties of cells and tissues are related to their structures and function and change of those properties can reflect certain pathology and also cellular healthy state. Providing physicians with quantitative elastography contrast would be a powerful aid for diagnostic. In the last decades, a large number of methods have been developed to combine quantitative elastography with biomedical imaging modalities [1-3].

Elastography has first been combined with optical systems in 1998 in OCT [4]. Since this pioneering work, a large number of methods were developed [3]. Recently, Nguyen et al [5] introduced in OCT a new passive shear wave elastography approach that overcame a major limitation of classical methods: the need of a well-controlled and wellsynchronized shear wave source. We present here an adaptation of this method for digital holography [6]. This approach allows to perform full field quantitative elastography using diffuse shear wave fields. The principle is to numerically refocus the shear wave field at each pixel using noise-correlation algorithms. The refocusing gives access to the local shear wave wavelength directly related to the local stiffness. Also, combining noise-correlation with full-field images allow the retrieval of two dimensional information of the medium's stiffness, which gives intrinsic access to anisotropy.

The experimental setup used for this study is a Mach-Zehnder-based off-axis digital holography system using a single mode LASER at $671 \mathrm{~nm}$ with 50x50 $\mu \mathrm{m}$ resolution over $11 \times 11 \mathrm{~mm}^{2}$ field-of-view. It allows to record shear wave propagation with a typical sensitivity of $50 \mathrm{~nm}$ at 500 frames/s.

For this study two imaging modes were implemented. The first mode consists in a stroboscopic approach. The camera and the mechanical source are synchronized to record a shear-wave propagation at high frame rate (equivalent to $25 \mathrm{kHz}$ ). With this mode, controlled experiments could be performed using time-of-flight. The second mode is a passive mode used to record diffuse displacement fields with no controlled shear-wave source. In this case, the acquired images are uncorrelated in time. Piezoelectric actuators were used as shear-wave sources. In the second imaging mode they created a consistent diffuse field at the surface of the sample for validation. In the second mode, only one actuator was activated to deliver a single impulse for propagation imaging.

We present here the validation of the noise-correlation method on polymer test sample with homogeneous optical properties and controlled mechanical heterogeneous properties that mimic biological tissues (Agarose and PVA, isotropic and anisotropic). Figure 1 shows some results on agarose inclusion samples, where the algorithm successfully retrieved soft inclusions. Figure 2 presents first results on a pig liver sample, where half of the sample was left raw as the other side was cooked. 

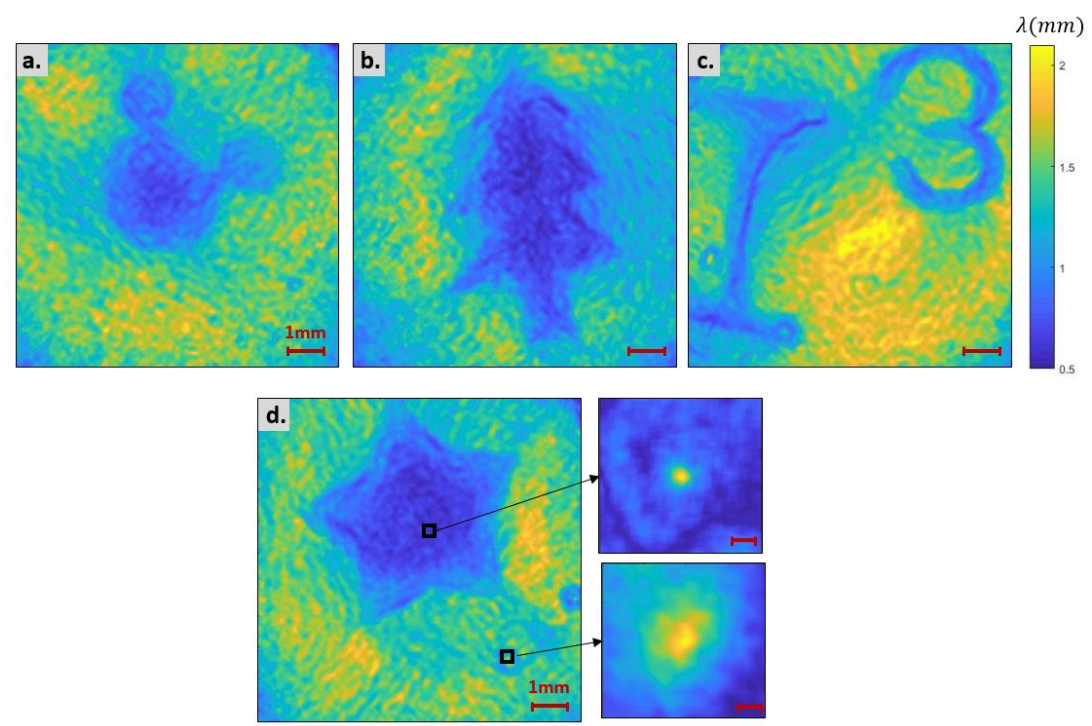

Figure 1: Shear wave wavelength map calculated from a diffuse shear wave field generated by piezoelectric actuators at a central frequency of $1.1 \mathrm{kHz}$. The samples a., b. c. and d. consist of soft $1 \%$ agarose inclusions in $2 \%$ stiffer agarose. The noise-correlation-based method was used to retrieve the quantitative stiffness map and the mechanical point spread functions have been traced for two points of the acquisition $\mathrm{d}$.

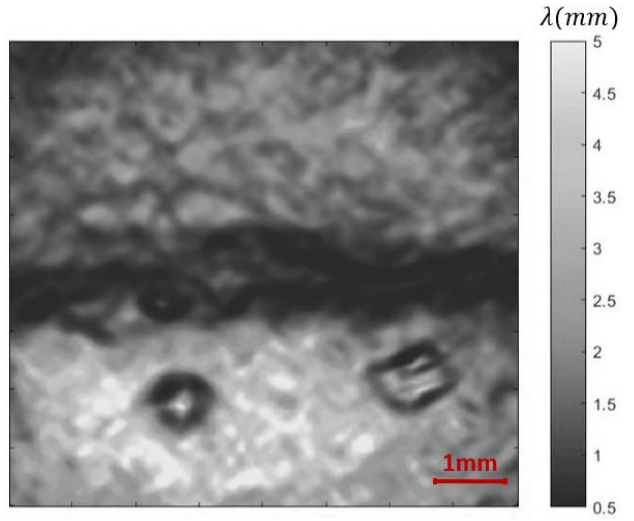

Figure 2: Quantitative wavelength map on a pork liver sample with using a noise-correlation (central frequency 1.5kHz). Half of the sample was left raw (top of the image) and half was cooked (below).

The noise-correlation approach for full-field elastography using digital holography is experimentally validated. This method tackles a strong optical shear-wave elastography limitation: the need of a controlled shear wave source, which currently limit the translation of this method into clinic. It is well adapted to full field coherent imaging techniques such as digital holography and full field OCT as the limiting factor is usually the low frame rate of the camera.

[1] Mariappan, Yogesh K., Glaser, Kevin J., et Ehman, Richard L. “Magnetic resonance elastography: a review”. Clinical anatomy, 2010, vol. 23, no 5, p. 497-511.

[2] Tanter, Mickael, Bercoff, Jeremy, Athanasiou, Alexandra, et al. "Quantitative assessment of breast lesion viscoelasticity: initial clinical results using supersonic shear imaging". Ultrasound in medicine \& biology, 2008, vol. 34, no 9, p. 1373-1386.

[3] Kennedy, Brendan F., Wijesinghe, Philip, et Sampson, David D. "The emergence of optical elastography in biomedicine." Nature Photonics, 2017, vol. 11, no 4, p. 215.

[4] Schmitt, Joseph M. "OCT elastography: imaging microscopic deformation and strain of tissue". Optics express, 1998, vol. 3, no 6, p. $199-211$. [5] Nguyen, Thu-Mai, Zorgani, Ali, Lescanne, Maxime, et al. "Diffuse shear wave imaging: toward passive elastography using low-frame rate spectral-domain optical coherence tomography". Journal of biomedical optics, 2016, vol. 21, no 12, p. 126013.

[6] Marmin, Agathe, Catheline, Stefan and Nahas, Amir "Full-field passive elastography using digital holography," Opt. Lett.45,2965-2968 (2020) 